\title{
Supporting Information: Twinning Organosuperelasticity of a Fluorinated Cyclophane Single Crystal
}

Toshiyuki Sasaki, Shunichi Sakamoto, Satoshi Takamizawa*

\begin{abstract}
:
Superelasticity is characterized by unique mechanical behavior, i.e., spontaneous shape recoverability by diffusionless plastic deformation and has been considered to be a property of specific alloys, so-called shape memory alloys, from its discovery in 1932. The discovery of superelasticity in an organic crystal in 2014, so-called organosuperelasticity, paved the way for functionalization of superelastic materials based on molecular design. In this context, achieving superelasticity using various kinds of organic compounds and elucidation of its mechanism are inevitable. Here we show superelasticity in a single crystal of a derivative of sterically bulky cyclophanes: 4,5,7,8,12,13,15,16-octafluoro[2.2]paracyclophane. Crystallographic studies indicated interconversion of two centrosymmetric crystallographically independent molecules by changing molecular orientations in the crystalline state in twinning superelasticity. Our findings will contribute to propose one of the useful approaches for designing organosuperelastic materials.
\end{abstract}


Table of Contents:

Page

(a) Experimental information $\quad$ S3

(b) Superelastic deformation $\quad \mathrm{S} 4$

$\begin{array}{lr}\text { (c) Crystallographic studies } & \text { S5-S7 }\end{array}$

(d) Supplementary references $\quad$ S8

Other supplementary materials:

Movie S1: Superelastic behavior of a $\mathbf{p} \mathbf{C} \mathbf{p}-\mathbf{F}_{\mathbf{8}}$ crystal at room temperature 


\section{(a) Experimental information}

\section{(Preparation of single-crystal samples)}

4,5,7,8,12,13,15,16-Octafluoro[2.2]paracyclophane (pCp-F $\mathbf{F}_{\mathbf{8}}$ ) was purchased from Tokyo Chemical Industry and toluene was purchased from Wako. Both were used as received. Wellformed single crystals were prepared by recrystallization of $\mathbf{p} \mathbf{C} \mathbf{p}-\mathbf{F}_{\mathbf{8}}$ from a concentrated solution in toluene.

\section{(Stress-strain test)}

Shear tests were carried out on a universal testing machine. Crystal were sheared by a glass jig attached to a load cell and observed under a polarization microscope coupled with a digital camera.

\section{(Single-crystal X-ray structure analysis)}

Single-crystal X-ray diffraction measurements of crystals of $\mathbf{p} \mathbf{C} \mathbf{p}-\mathbf{F}_{\mathbf{8}}$ were performed at $298 \mathrm{~K}$ $\left(25^{\circ} \mathrm{C}\right)$ on a Bruker Smart APEX diffractometer equipped with CCD area detector and a nitrogenflow temperature controller using graphite-monochromated MoK $\alpha$ radiation $(\lambda=0.71073 \AA)$. Multi-scan absorption corrections were applied using the SADABS program. ${ }^{\mathrm{S1}}$ The structure was solved by intrinsic phasing methods (SHELXT-2014/5) ${ }^{\mathrm{S2}}$ and refined by full-matrix least-squares calculations on $F^{2}$ (SHELXL-2014/7). ${ }^{\mathrm{S} 3}$ Non-hydrogen atoms were refined anisotropically; hydrogen atoms were fixed at calculated positions by riding model approximation. Crystallographic data of the structures are summarized in Table S2. Crystal face indexing was performed using SMART ${ }^{\text {S4 }}$ in a SHELXL Ver. 6.12 program package. Miller plane interplanar angles were measured using Mercury CSD 3.9. ${ }^{\mathrm{S} 5}$ 


\section{(b) Superelastic deformation}

Table S1. Comparison of mechanical properties among organosuperelastic materials.

\begin{tabular}{ccccccc}
\hline Entry & $\sigma_{\mathrm{f}} / \mathrm{MPa}^{a}$ & $\sigma_{\mathrm{r}} / \mathrm{MPa}^{a}$ & $E_{\mathrm{s}} / \mathrm{kJ} \mathrm{m}^{-3 b}$ & $\eta^{c}$ & $\chi^{d}$ & $\varepsilon_{\max } / \%^{e}$ \\
\hline${\mathrm{pCp}-\mathrm{F}_{8}{ }^{f}}^{0}$ & 0.346 & 0.029 & 8.2 & 0.131 & 0.04 & 29.2 \\
$\mathrm{TPA}^{g}$ & 0.496 & 0.459 & 62.0 & 0.925 & 0.13 & 11.3 \\
$3,5 \mathrm{DFB}^{h}$ & 0.070 & $0.01-0.03$ & 11.9 & 0.286 & 0.26 & 52.7 \\
\hline
\end{tabular}

${ }^{a}$ Effective shear stress for deformation: Proceeding in the forward $\left(\sigma_{\mathrm{f}}\right)$ and reverse $\left(\sigma_{\mathrm{r}}\right)$ directions.

${ }^{b}$ Energy storage density $\left(E_{s}=W_{\text {out }} / V\right)$. The symbols $W_{\text {out }}$ and $V$ represent the output work and volume, respectively, in a deformed region of a specimen during superelastic deformation. ${ }^{c}$ Energy storage efficiency $\left(\eta=W_{\text {out }} / W_{\text {in }}\right)$ where $W_{\text {in }}$ represents input work. ${ }^{d}$ Superelastic index $\left(\chi=2 E_{s} /\left(\sigma_{\mathrm{f}}+\sigma_{\mathrm{r}}\right)\right)$. ${ }^{e}$ Maximum shear strain $\left(\varepsilon_{\max }=\tan \theta\right)$ where $\theta$ is bending angle.

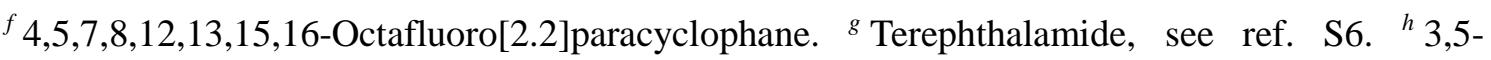
Difluorobenzoic acid, see ref. S7. 


\section{(c) Crystallographic studies}
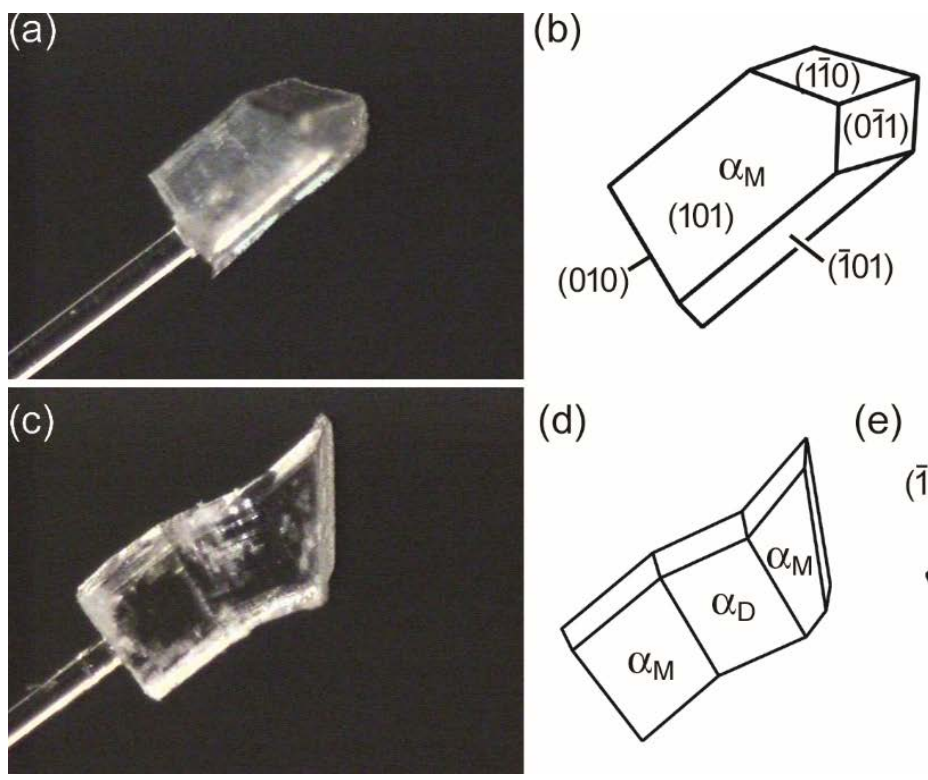

(d)

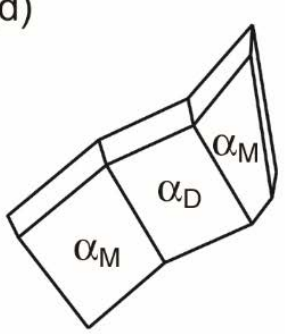

(e)

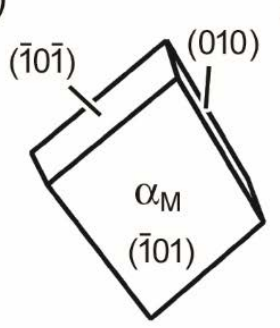

(f) (101)

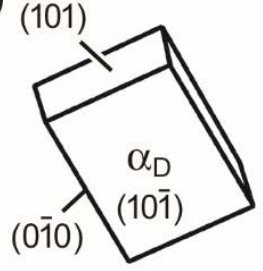

Figure S1. Face indexing of $\mathbf{p} \mathbf{C} \mathbf{p}-\mathbf{F}_{\mathbf{8}}$ crystals. (a) A crystal photo of an as-prepared $\mathbf{p} \mathbf{C} \mathbf{p}-\mathbf{F}_{\mathbf{8}}$ crystal, (b) its schematic representation and face indexing. (c) A crystal photo of a twinned $\mathbf{p} \mathbf{C} \mathbf{p}-\mathbf{F}_{\mathbf{8}}$ crystal, (d) its schematic representation, and (e) face indexing of the $\alpha_{M}$ domain and (f) $\alpha_{D}$ domain.
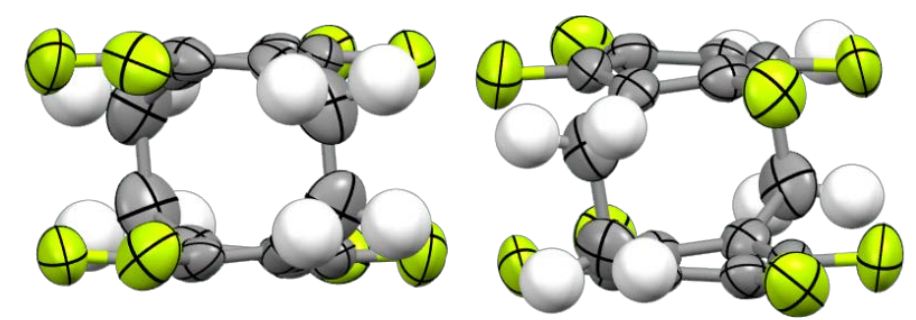

Figure S2. Independent molecules: Z1 (left) and Z2 (right), in the asymmetry unit of a $\mathbf{p} \mathbf{C p}_{\mathbf{p}} \mathbf{F}_{\mathbf{8}}$ crystal represented by thermal ellipsoids at the $50 \%$ probability level extracted from the crystal structure of the as-prepared crystal. 
(a)

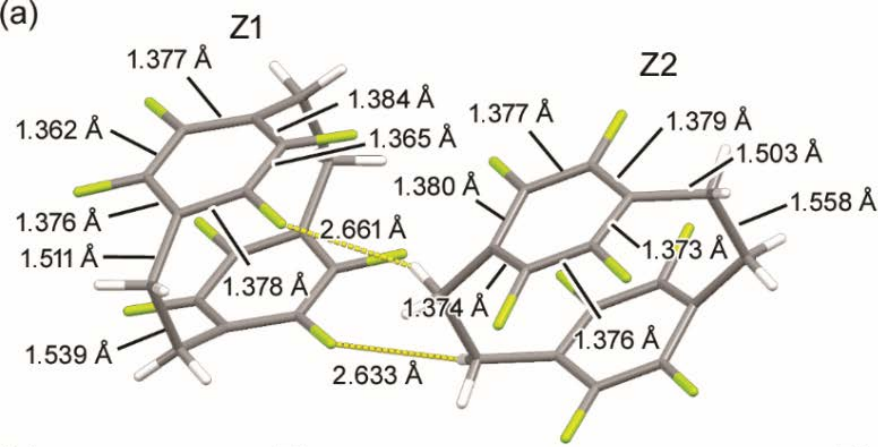

(b)
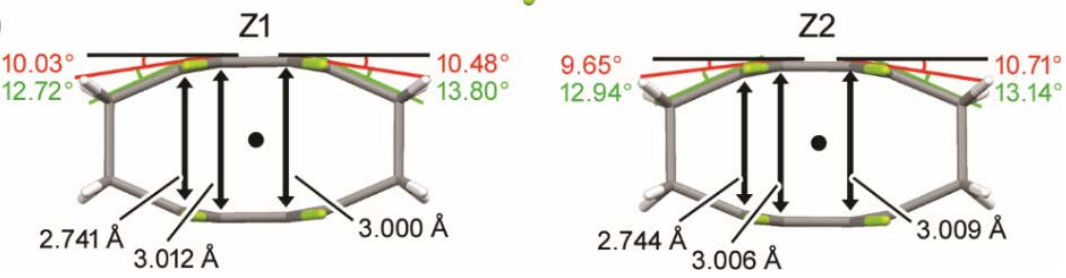

Figure S3. (a) Lengths of covalent bonds of Z1 and Z2 and distances of $\mathrm{H} \cdots$ F hydrogen bond interactions between Z1 and Z2. (b) Deviation angles of benzene rings and C-C distances between the confronted benzene rings in Z1 and Z2 molecules. Black circles represent inversion centers. Those values are measured in the crystal structure analyzed using the as-prepared OFPCP crystal.

(a)
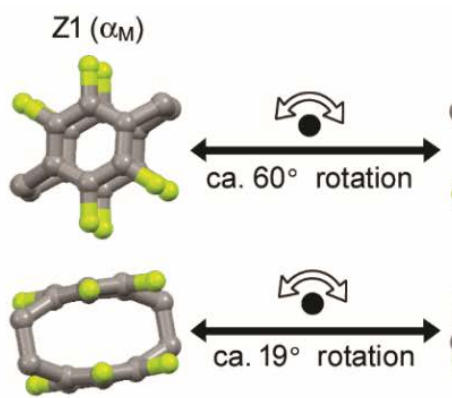

(c)

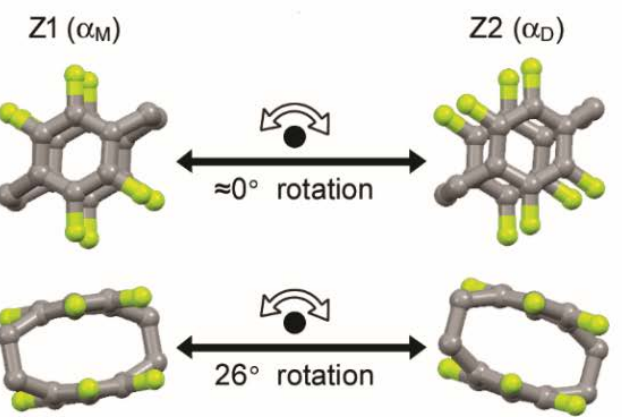

(b) $\quad \mathrm{Z} 2\left(\alpha_{M}\right)$
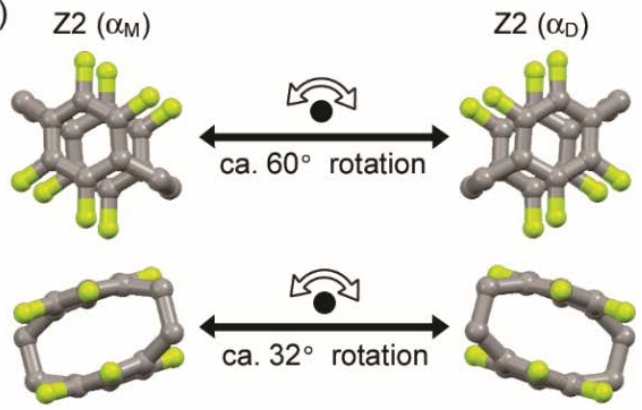

(d)

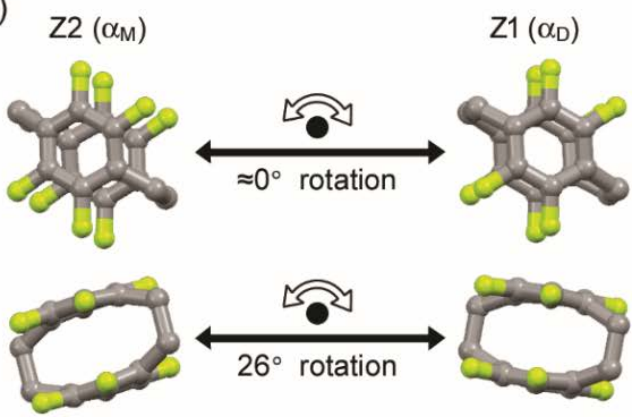

Figure S4. Possible molecular rotations of $\mathbf{p} \mathbf{C p}_{\mathbf{p}} \mathbf{F}_{\mathbf{8}}$ during superelastic deformation between (a) Z1 ( $\left.\alpha_{M}\right)$ and Z1 $\left(\alpha_{D}\right)$, (b) Z2 ( $\left.\alpha_{M}\right)$ and Z2 ( $\left.\alpha_{D}\right)$, (c) Z1 ( $\left.\alpha_{M}\right)$ and Z2 $\left(\alpha_{D}\right)$, and (d) Z2 ( $\left.\alpha_{M}\right)$ and Z1 $\left(\alpha_{D}\right)$. Rotation axes are the intersections of the (-101) $\alpha_{M}-(-10-1) \alpha_{M}$ (top) and $(010) \alpha_{M}-(-10-1) \alpha_{M}$ (bottom). 
Table S2. Crystallographic data of $\mathbf{p} \mathbf{C} \mathbf{p}-\mathbf{F}_{\mathbf{8}}$ : as-prepared crystal, mother and daughter domains in a superelastically deformed crystal.

\begin{tabular}{|c|c|c|c|}
\hline Entry & As-prepared & Mother & Daughter \\
\hline Empirical formula & $\mathrm{C}_{16} \mathrm{H}_{8} \mathrm{~F}_{8}$ & $\mathrm{C}_{16} \mathrm{H}_{8} \mathrm{~F}_{8}$ & $\mathrm{C}_{16} \mathrm{H}_{8} \mathrm{~F}_{8}$ \\
\hline M & 352.22 & 352.22 & 352.22 \\
\hline Crystal shape & block & block & block \\
\hline Crystal size $/ \mathrm{mm}^{3}$ & $0.890 \times 0.475 \times 0.306$ & $0.501 \times 0.312 \times 0.194$ & $0.534 \times 0.395 \times 0.178$ \\
\hline Crystal system & Triclinic & Triclinic & Triclinic \\
\hline Space group & $P-1$ & $P-1$ & $P-1$ \\
\hline$T / \mathrm{K}$ & 298 & 298 & 298 \\
\hline$a / \AA$ & $7.759(3)$ & $7.741(5)$ & $7.752(6)$ \\
\hline$b / \AA$ & $8.316(3)$ & $8.304(5)$ & $8.321(6)$ \\
\hline c /Å & $10.470(4)$ & $10.463(6)$ & 10.473(8) \\
\hline$\alpha / \operatorname{deg}$ & $94.731(5)$ & $94.749(9)$ & $94.716(10)$ \\
\hline$\beta /$ deg & 95.339(5) & $95.309(8)$ & $95.310(11)$ \\
\hline$\gamma /$ deg & $96.179(5)$ & 96.156(9) & $96.184(11)$ \\
\hline$V / \AA^{3}$ & $665.8(4)$ & $662.9(7)$ & $665.9(9)$ \\
\hline Z & 2 & 2 & 2 \\
\hline $\mathrm{D}_{\text {calcd }} / \mathrm{Mg} \mathrm{m}^{-3}$ & 1.757 & 1.765 & 1.757 \\
\hline$\mu(\mathrm{Mo} \mathrm{K} \alpha) / \mathrm{mm}^{-1}$ & 0.181 & 0.181 & 0.181 \\
\hline Reflections collected & 3174 & 3099 & 3084 \\
\hline Independent reflections $\left(\mathrm{R}_{\text {int }}\right)$ & $2289(0.0378)$ & $2247(0.0473)$ & $2256(0.0633)$ \\
\hline Goodness of fit & 0.997 & 1.445 & 1.960 \\
\hline $\mathrm{R}_{1}(\mathrm{I}>2 \sigma$ (all data) $)$ & $0.0967(0.1050)$ & $0.0928(0.1080)$ & $0.1464(0.1597)$ \\
\hline $\mathrm{wR}_{2}(\mathrm{I}>2 \sigma$ (all data $\left.)\right)$ & $0.2222(0.2289)$ & $0.3122(0.3193)$ & $0.4126(0.4178)$ \\
\hline Largest diff. peak (hole) /e $\AA^{3}$ & $0.797(-0.539)$ & $0.382(-0.361)$ & $0.736(-0.620)$ \\
\hline CCDC No. & 1939997 & 1939998 & 1939999 \\
\hline
\end{tabular}




\section{(d) Supplementary references}

[S1] SADABS, Bruker AXS Inc., Madison, Wisconsin, USA.

[S2] Sheldrick, G. M. SHELXT - Integrated Space-Group and Crystal-Structure Determination. Acta Cryst. 2015, A71, 3-8.

[S3] Sheldrick, G. M. Crystal Structure Refinement with SHELXL. Acta Cryst. 2015, C71, 3-8.

[S4] SMART, Bruker AXS Inc., Madison, Wisconsin, USA.

[S5] Macrae, C. F.; Bruno, I. J.; Chisholm, J. A.; Edgington, P. R.; McCabe, P.; Pidcock, E.; Rodriguez-Monge, L.; Taylor, R.; van de Streek, J.; Wood, P. A. Mercury CSD 2.0 - New Features for the Visualization and Investigation of Crystal Structures. J. Appl. Cryst. 2008, 41, 466-470.

[S6] Takamizawa, S.; Miyamoto, Y. Superelastic Organic Crystals. Angew. Chem. Int. Ed. 2014, 53, 6970-6973.

[S7] Takamizawa, S.; Takasaki, Y. Superelastic Shape Recovery of Mechanically Twinned 3,5Difluorobenzoic Acid Crystals. Angew. Chem. Int. Ed. 2015, 54, 4815-4817. 